\title{
Sonographic description and classification of tendinous involvement in relation to tophi in chronic tophaceous gout
}

\author{
Eloy de Ávila Fernandes • Gabriel B. Sandim • \\ Sônia A. V. Mitraud • Edson S. Kubota • \\ Antonio J. L. Ferrari • Artur R. C. Fernandes
}

Received: 11 April 2010 /Revised: 7 June 2010 / Accepted: 21 June 2010 /Published online: 4 July 2010

(C) European Society of Radiology 2010

\begin{abstract}
Objective To describe and classify the varied ultrasound features of tendinous involvement in relation to tophi in chronic tophaceous gout so that they are better recognised. Methods Ultrasound images of 138 affected areas from 31 patients with chronic tophaceous gout were analysed using high-quality broadband linear transducers. The relationship between tendon and tophi was classified, and the interobserver agreement regarding classification was analysed. Results Tophi envelopment in the tendon was the most frequent characteristic (45\%) followed by no relationship between tophi and tendon (41\%), tophi at the insertion site of the tendon (7\%), extrinsic compression (6\%) and tophi within the tendon (1\%). The inter-observer concordance on classification of the relationship between tophi and tendon was measured using McNemar's test with $P<0.001\left(\chi^{2}=\right.$ 30.0 , degree of freedom $=9)$ and kappa test $=0.627(P<$ 0.001 ), indicating substantial inter-observer concordance. Conclusion Tophi generally envelope the tendon or there is no relationship between them. Tophi can also be found at the insertion site of the tendon, cause extrinsic compression or be located inside the tendon. There is substantial interobserver agreement for ultrasound classification of tendon involvement by tophi. This study contributes to diagnostic
\end{abstract}

E. de Ávila Fernandes $(\bowtie) \cdot$ G. B. Sandim • S. A. V. Mitraud •

E. S. Kubota $\cdot$ A. R. C. Fernandes

Diagnostic Imaging Department,

Federal University of São Paulo - UNIFESP,

Rua Napoleão de Barros, 800, térreo,

CEP: 04024-002 São Paulo, SP, Brasil

e-mail: lofernan@uol.com.br

\section{A. J. L. Ferrari}

Rheumatology Discipline of Medicine Department,

Federal University of São Paulo,

São Paulo, Brazil elucidation and shows the diverse characteristic forms of tendon involvement by tophi.

Keywords Gout · Arthritis · Tendon injuries · Ultrasound . Diagnosis

\section{Introduction}

Gout is an inflammatory, metabolic illness that occurs in conjunction with hyperuricaemia and is a result of the deposition of monosodium urate crystals (MSU) mostly in soft tissues and the joints of extremities. It is a relatively common disease, occurring worldwide in 0.2 to 0.35 per 1,000 people in the general population. The highest incidence is seen at the end of the third and beginning of the fourth decades of life, with predominance in the male sex ( $95 \%$ of the cases). In females it generally occurs after the menopause.

Chronic tophaceous gout is the clinical presentation that is manifested by the presence of the tophus which is associated with the deposition of MSU, protein matrix, inflammatory cells and foreign body giant cells in the tissues [1] and which occurs after between 5 and 12 years of inadequately treated illness.

Local anatomical deformities can be caused by tophi infiltration into structures such as tendons [2, 3] with consequent limitation or loss of articular movements. It was believed that gouty tophi did not lead to tendon destruction, one important difference from pannus. However, after cases reported in the literature that show tendon ruptures and surgical findings of a thick, chalky-white substance consistent with gouty tophus, embedded within tendons [4], and tendon ruptures where massive white crystals were deposited intratendinously and paratendinously [5], it is 
now accepted that gouty tophi may play a role in tendon destruction.

Considering that ultrasound has recently been identified as a promising new imaging technique for gout, the aim of this study is to describe and classify the different ultrasound features of tendinous involvement by tophi in chronic tophaceous gout so that they may be recognised by the sonographer and assist the physician in the management of the disease.

It is necessary to evaluate the feasibility of this classification to verify if these aforementioned features, related to tendinous involvement of tophi in chronic tophaceous gout, could be applied by other physicians. Analysis of inter-observer concordance was used to address this question.

\section{Materials and methods}

A total of 31 adult patients diagnosed as having tophaceous gout by the Microcrystal Disease Clinic of the Discipline of Rheumatology of the Federal University of São Paulo UNIFESP were referred to us for further evaluation with appropriate diagnostic imaging from October 2005 to December 2008, with a total of 138 affected areas analysed showing visible elevation of the skin surface. Information concerning the research was provided to all the participants, and they all signed a free and informed consent form approved by the institutional ethics committee of UNIFESP.

The inclusion criteria for this study were as follows: patients of both sexes older than 21 years diagnosed with gout by the discovery of crystals in synovial fluid, patients who met the ACR [6] criteria for gout and patients with chronic tophaceous gout with or without chronic renal insufficiency. The exclusion criteria were patients who presented with gout but without tophus and patients with other crystal illnesses. The age of patients studied ranged from 33 to 77 years, with an average of 56.5 years. There were 31 men and no women. The duration of the illness cited by the patients ranged from 4 to 30 years (median 12.32 years). The ultrasound images of these areas were obtained using high-quality broadband linear transducers with a frequency range of $8-14 \mathrm{MHz}$ (Sonoline Antares, Siemens, Munich, Germany and EnVisor Philips, Bothell, USA). All ultrasound images were collected following a previously determined protocol at the Radiology Department of UNIFESP, where all well-defined tophi that were present on visual inspection as abnormal skin protuberances and that could be measured by ultrasound were analysed with imaging in the longitudinal and cross-sectional planes in the major axes of the visible nodulation. The ultrasound features of tophi were characterised by the presence of bright spots and hyperechoic areas [7].
The relationship between tophi and tendons was classified as follows: A: tophi enveloping the tendon, B: no relationship between the tophi and the tendon, $\mathrm{C}$ : tophi at the insertion site of the tendon, D: extrinsic compression of the tendon, and E: tophi inside the tendon.

The examinations were carried out by a single musculoskeletal radiologist (E.A.F.) with 15 years' experience in musculoskeletal ultrasound. All the ultrasound images were later reviewed together with a third-year resident of radiology (G.B.S.). The cases in which there was disagreement were resolved in consensus.

To analyse the inter-observer agreement regarding the relationship between tophi and the tendon, another musculoskeletal radiologist (S.V.) with 15 years' experience in musculoskeletal ultrasound carried out a new analysis of the static images. The data relative to the relationship between tophi and the tendon were analysed using the kappa and McNemar-Bowker tests. The strength of interobserver agreement was rated following the recommendation of Landis and Koch. Differences were considered significant when $P<0.05$. [8].

\section{Results}

The age of the patients studied ranged from 33 to 77 years, with an average of 56.5 years. There were 31 men and no women. The duration of illness cited by the patients ranged from 4 to 30 years (median 12.32 years). Different areas were affected by tophi (Table 1 ).

The characteristics of the relationship between tophi and tendon are shown in Table 2. Tendon enveloped by tophi was the most frequent feature, observed in 62 areas. In 56 areas there was no relationship between tophi and the tendon, in 10 areas tophi were located at the insertion site of the tendon, in 9 areas there was extrinsic compression and in 1 area a tophus was found inside the tendon. Figures 1, 2, 3, 4 and 5 illustrate these features.

There was substantial inter-observer agreement relative to ultrasound features of tendinous involvement by the tophi in chronic tophaceous gout through the application of

Table 1 Localisation and number of areas of tophi analysed in patients with chronic tophaceous gout

\begin{tabular}{lll}
\hline Localisation of nodules & Number & Percentage \\
\hline Ankle, foot & $49 / 138$ & 36 \\
Hand, wrist & $39 / 138$ & 28 \\
Forearm, elbow & $37 / 138$ & 27 \\
Knee, leg & $8 / 138$ & 6 \\
Auricular appendage & $5 / 138$ & 3 \\
\hline
\end{tabular}


Table 2 Classification of the relationship between tophi and the tendon in chronic tophaceous gout

\begin{tabular}{llll}
\hline Types & Relation to tendon & Number & Percentage \\
\hline A & Enveloped & $62 / 138$ & 45 \\
B & No relation & $56 / 138$ & 41 \\
C & Insertion site & $10 / 138$ & 7 \\
D & Extrinsic compression & $9 / 138$ & 6 \\
E & Inside & $1 / 138$ & 1 \\
\hline
\end{tabular}

McNemar-Bowker test (chi-squared equal to 30.0 with $P<0.001)$ and a kappa index of $0.627(P<0.001)$.

\section{Discussion}

Tophi envelopment of the tendon was the most common characteristic of the relationship between tophi and the tendon, seen in 62/138 cases (45\%) of the analysed areas, which were classified as type A. Tophi envelopment may occur because the microcrystal deposition begins initially from the external part of the tendon such as in the articular cartilage where the deposits are located within the superficial layers [9].

The tendinous involvement by tophi in chronic tophaceous gout has been referred to using different terms in
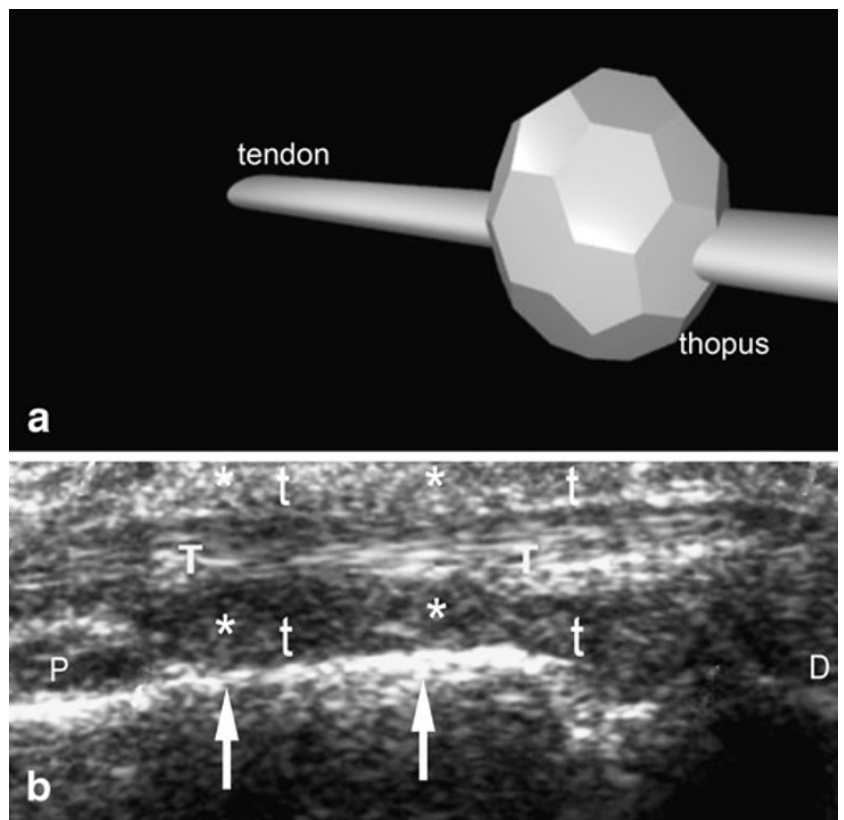

Fig. 1 a Computer-generated image of a tophus enveloping the tendon. b Ultrasound image of the dorsal aspect of the extensor tendon (T) of the hand in the longitudinal plane ( $D$ distal, $P$ proximal), showing the multiple bright stippled foci of the tophus (asterisk and $t$ ), enveloping the tendon $(T)$ with blurring of the contours of the tendon. Arrows mark the bone surface of the adjacent metacarpal bone

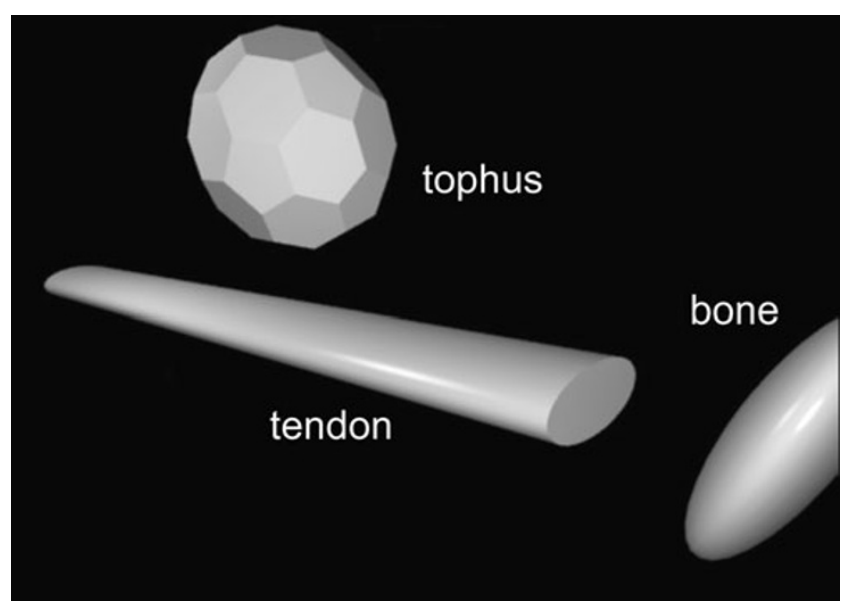

Fig. 2 Computer-generated image: no relation between tophi and tendon

the literature. The term "intratendinous infiltration" includes many aspects of the relationship between tophi and the tendon, as seen in reports that show that gout can affect the tendons through intratendinous infiltration $[10,11]$ causing compressive syndromes [12-14]. The term "infiltration" can encompass tophi within the tendon, those leading to extrinsic compression and those enveloping the tendon. For this reason the more specific characterisation of the relationship between tophi and tendon may
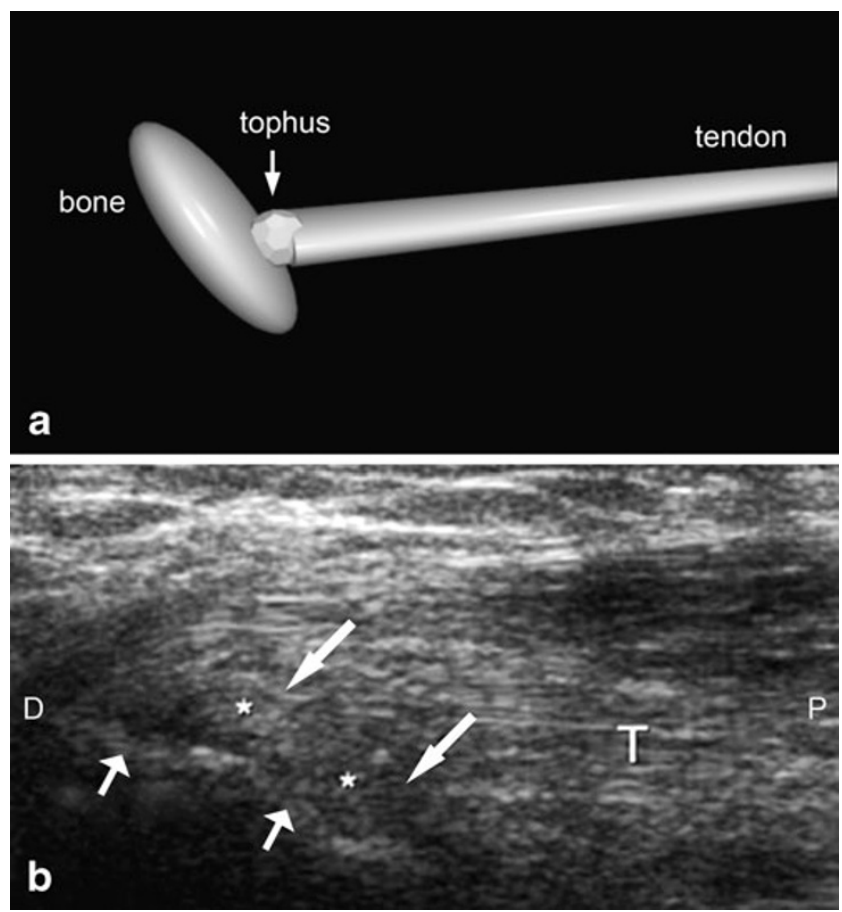

Fig. 3 a Computer-generated image of tophi located at the insertion site of the tendon adjacent to the bone. b Ultrasound image in the longitudinal plane ( $D$ distal, $P$ proximal) showing tophi (asterisk and arrows) seen as circular hyperechoic areas with bright stippled foci at the insertion site of the triceps tendon $(T)$ of the elbow, which has loss of fibrillary pattern close to the bone (olecranon, small arrows) 



Fig. 4 a Computer-generated image of a tophus causing extrinsic compression on the tendon. b Longitudinal ultrasound image $(P$ proximal, $D$ distal) of the lateral aspect of the ankle showing tophus causing extrinsic compression (arrowheads) on the peroneus brevis tendon $(T)$. Note the hyperechoic tophus under the surface of the tendon, causing a slight compressive effect (arrowheads). Arrows mark the bone surface of the talus

assist with the topographic pathological aspects, facilitate the differential diagnosis and therefore greatly increase efficient therapeutic follow-up with ultrasound imaging of the patients.

Sometimes gouty tendon involvement can present as pain and local warmth that is often difficult to tell apart from infection [2, 11, 15], and some authors advocate diagnostic imaging with high-resolution ultrasound and needle aspiration of the peritendinous fluid, which can change the course of treatment [16]. In some cases, these symptoms may be related to tophi envelopment of tendons (type A) and the recognition of the ultrasound features of this envelopment can help to reduce the need for invasive procedures, confirm the diagnosis and enable adequate treatment.

The precise characterisation of the relation between the tophi and the tendons can help in the definition of pathological process before the cutaneous tophus manifestation or elevation of the skin, and can also make the diagnosis more consistent so that treatment can be started earlier.
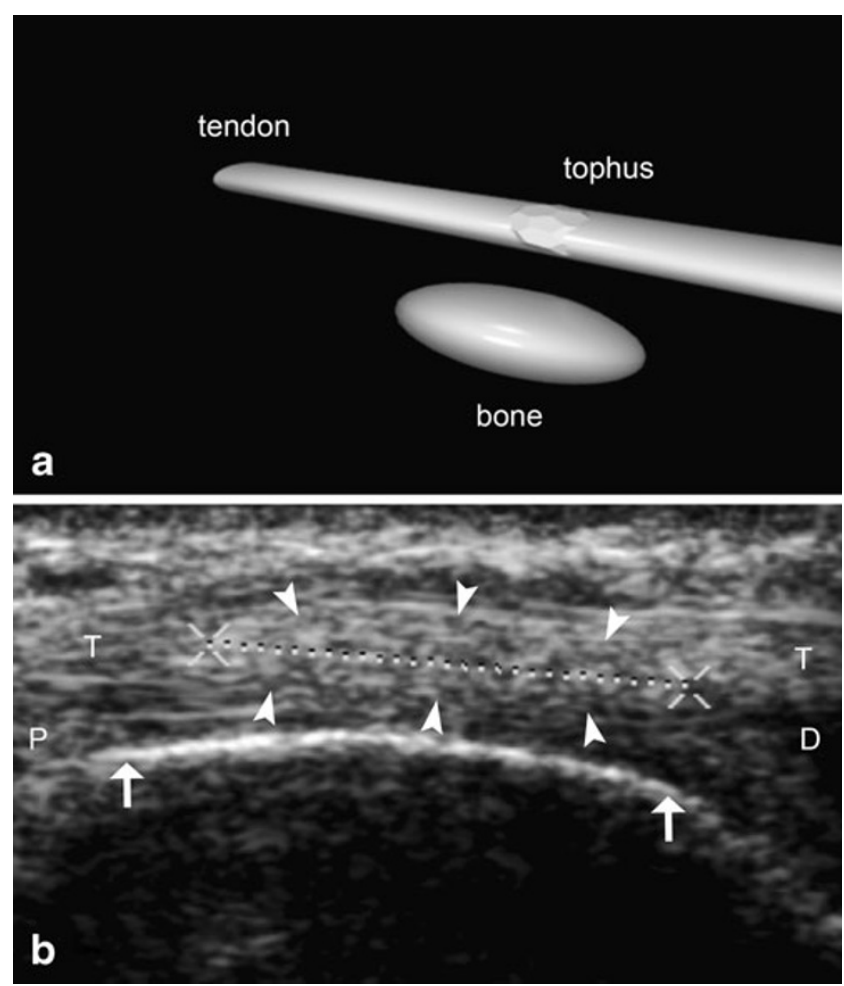

Fig. 5 a Computer-generated image of tophus inside the tendon. b Longitudinal ultrasound image ( $P$ proximal, $D$ distal) of the distal forearm (radius, arrows) showing a cross-section of the tophus (dotted line between Xs and arrowheads) inside the extensor pollicis longus tendon $(T)$ resulting in loss of its fibrillary pattern

The importance of the relationship between intratendinous tophi and envelopment of tendons and their subsequent evolution to longitudinal ruptures as a cause of relevant symptoms has already been observed in a case report described by Lagoutaris et al. [4]. When dealing with similar cases, knowing the ultrasound aspects of tophus enveloping the tendon (type A) and tophus inside the tendon (type E), it is possible to indicate clinical treatment to reduce the dimensions of tophi in order to avoid rupture of the tendon and surgery.

In $56 / 138$ cases $(41 \%)$ of affected areas, type B was found, where there was no relationship between the tophi and the tendon, i.e. the tophi had no contact whatsoever with the tendon. This may be explained by the fact that urate deposition also occurs in other places besides tendons such as in the articular cartilage, subchondral bone, synovial membrane, capsular and periarticular tissues, and in areas of decreased temperatures such as in more superficial tissues of the extremities.

Type $\mathrm{C}$ was found in $10 / 138$ areas $(7 \%)$ where tophi were located at the insertion site of the tendon, in direct contact with both the tendon and the connecting bone, characterising enthesopathy. The differential diagnoses of enthesopathies are varied and may include degenerative disease, 
acromegaly, ankylosing spondylitis, diffuse idiopathic skeletal hyperostosis, calcium pyrophosphate dehydrate crystal deposition, hyperparathyroidism, hypoparathyroidism, juvenile chronic arthritis, Lyme's disease, rheumatoid arthritis and other diseases. The number of enthesopathies found in this study (7\%) is an important finding as, to our knowledge, there is only one reported case in which tophi at the insertion site of the tendon were considered a cause of enthesopathy [17], and as a result of this finding, chronic tophaceous gout should be considered in the differential diagnosis of enthesopathy.

Type $\mathrm{D}$, in which there was extrinsic compression on the tendon by the tophi, was found in $9 / 138$ areas $(6 \%)$ and can be considered a peritendinous lesion. There is great difficulty in diagnosing peritendinous lesions due to the various differential diagnoses, which include ganglion, Baker's cyst, tenosynovitis, bursitis, haematoma, amyloidosis, thrombophlebitis and other tumour or tumour-like lesions as well as gout [18]. Knowledge of both forms (type A, tophi envelopment of the tendon, and D, extrinsic compression on the tendon), describing the relationship between tophi and tendon, can help the sonographer in the differential diagnosis of these conditions.

Type E, tophus inside the tendon, was found in $1 / 138$ areas $(1 \%)$. There is a possibility that the deposition of MSU in this rare case originally began within the tendon or that the outer portion of the tophus had been reduced by the effects of the treatment and that the remaining part of the tophus was left inside the tendon.

There is also difficulty in diagnosing lesions found inside the tendons as exemplified in two case reports where tophi in the rotator cuff were not diagnosed in imaging examinations $[19,20]$.The ultrasound and/or MRI images demonstrated lesions in the supraspinatus tendon that were considered rupture and calcific tendinosis. Arthroscopic and surgical findings demonstrated tophus inside the tendons, showing the difficulty in recognising the tophus by imaging methods and highlighting the possibility of considering gouty tophi in differential diagnosis of impingement syndrome in patients with chronic tophaceous gout. There is another case report in which ultrasound of the finger showed increased echogenicity and heterogeneity in the tendon. The patient was treated with oral non-steroidal anti-inflammatory medication without clinical improvement. After a few months, surgical treatment was performed and showed chalky, crystalline deposits on the surface of the tendon. Histopathology showed fragmented nodules with amorphous basophilic material resembling sodium urate deposits surrounded by palisaded layers of histiocytes and occasional multinucleate giant cells typical of gouty tophus [3]. Although a tophus was found inside the tendon in only one case in the present study, it is possible that this incidence would be higher if all tendons were analysed in patients with chronic tophaceous gout.
To show the reproducibility of the relationship features between tophi and tendon, the data were analysed and indicated a substantial inter-observer agreement between the radiologists, with a kappa index result of $0.627(P<$ 0.001 ) and McNemar-Bowker test (chi-squared equal to 30.0 with $P<0.001$ ). To our knowledge, there are no studies in the literature that describe this agreement in patients with proven tophaceous gout.

The limitations of this study are the lack of a gold standard method for comparison of ultrasound findings, the fact that we did not carry out clinical correlation, the small number of patients in the study and the lack of Doppler study of the nodules. Doppler and power Doppler ultrasound images could also be useful in the differentiation of gout from other inflammatory diseases, but, to give true validation to this affirmation, standard parameters would have to be established for the Doppler settings in different machines and for intra- and inter-observer analyses with the same machine and different machines, and new studies on this subject may be needed. The lack of a gold standard may be justified by the inclusion criteria (presence of crystals in the synovial fluid) and because the definitive proof of tophi would be the surgical extraction of the nodules; however, this invasive method is not justified as a routine, seeing as the use of medication is effective in reducing tophi.

The first step in the differentiation of tophi in gout from other rheumatic-inflammatory and crystal-deposit diseases must be the precise description and better characterisation of ultrasound images of tophi in patients with chronic tophaceous gout and the adoption of a classification system, as this is not clearly defined in the literature. The main purpose of this study was to develop a feasible classification system for tophi and their relationship with tendons.

Frequently clinical evaluation alone cannot fully explain the problems caused by tophi. By means of ultrasound examination of patients with chronic tophaceous gout, it was possible to clearly identify diverse forms of the relationship between the tendon and tophi and to attain substantial inter-observer agreement regarding ultrasound features. We are proposing a classification of this relationship that is reliable and may be easily recognised. This may assist in the diagnosis and in the management of the treatment of patients with chronic tophaceous gout.

\section{References}

1. Monu JU, Pope TL Jr (2004) Gout: a clinical and radiologic review. Radiol Clin North Am 42:169-184. doi:10.1016/S00338389(03)00158-1

2. Wurapa RK, Zelouf DS (2002) Flexor tendon rupture caused by gout: a case report. J Hand Surg Am 27:591-593. 
3. Coombs PR, Houseman N, White R (2006) Chronic tophaceous gout of the third flexor digitorum profundus tendon in the hand: an unusual sonography diagnosis. AJR Am J Roentgenol 187: W313-W315. doi:10.2214/AJR.05.0336

4. Lagoutaris ED, Adams HB, DiDomenico LA, Rothenberg RJ (2005) Longitudinal tears of both peroneal tendons associated with tophaceous gouty infiltration. A case report. J Foot Ankle Surg 44:222-224

5. Hung JY, Wang SJ, Wu SS (2005) Spontaneous rupture of extensor pollicis longus tendon with tophaceous gout infiltration. Arch Orthop Trauma Surg 125:281-284. doi:10.1007/s00402004-0670-9

6. Wallace SL, Robinson H, Masi AT, Decker JL, McCarty DJ, Yu TF (1977) Preliminary criteria for the classification of the acute arthritis of primary gout. Arthritis Rheum 20:895-900

7. Rettenbacher T, Ennemoser S, Weirich H, Ulmer H, Hartig F, Klotz W, Herold M (2008) Diagnostic imaging of gout: comparison of high-resolution US versus conventional X-ray. Eur Radiol 18:621-630. doi:10.1007/s00330-007-0802-z

8. Kundel HL, Polansky M (2003) Measurement of observer agreement. Radiology 228:303-308

9. Resnick DN (1988) Gouty arthritis. In: Resnick D (ed) Diagnosis of bone and joint disorders. Saunders, Philadelphia, pp 1618 1671

10. Primm DD Jr, Allen JR (1983) Gouty involvement of a flexor tendon in the hand. J Hand Surg Am 8:863-865
11. Moore JR, Weiland AJ (1985) Gouty tenosynovitis in the hand. J Hand Surg Am 10:291-295

12. Mockford BJ, Kincaid RJ, Mackay I (2003) Carpal tunnel syndrome secondary to intratendinous infiltration by tophaceous gout. Scand J Plast Reconstr Surg Hand Surg 37:186-187

13. Champion D (1969) Gouty tenosynovitis and the carpal tunnel syndrome. Med J Aust 1:1030-1032

14. Janssen T, Rayan GM (1987) Gouty tenosynovitis and compression neuropathy of the median nerve. Clin Orthop Relat Res 216:203-206

15. Townshend D, Pai V (2004) Gouty tenosynovitis-more common than we think? N Z Med J 117:U749

16. Nadim A, Lo S, McNab I (2004) Gouty flexor tenosynovitis mimicking infection: a case report emphasizing the value of ultrasound in diagnosis. Acta Orthop Belg 70:368-370

17. Bond JR, Sim FH, Sundaram M (2004) Radiologic case study. Gouty tophus involving the distal quadriceps tendon. Orthopedics 27(18):90-92

18. Ho CF, Chiou HJ, Chou YH, Chang CY (2003) Peritendinous lesions: the role of high-resolution ultrasonography. Clin Imaging 27:239-250.

19. O'Leary ST, Goldberg JA, Walsh WR (2003) Tophaceous gout of the rotator cuff: a case report. J Shoulder Elbow Surg 12:200-201. doi:10.1067/mse.2003.24

20. Chang CH, Lu CH, Yu CW, Wu MZ, Hsu CY, Shih TT (2008) Tophaceous gout of the rotator cuff. A case report. J Bone Joint Surg Am 90:178-182. 